\title{
TARGET DETECTION ALGORITHM FOR AIRBORNE THERMAL HYPERSPECTRAL DATA
}

\author{
Richa Marwaha, Anil Kumar, P. L. N. Raju and Y. V. N. Krishna Murthy \\ Indian Institute of Remote Sensing, (ISRO) Dehradun, India \\ (richamarwaha12@gmail.com, anil@iirs.gov.in, raju@iirs.gov.in, director@iirs.gov.in)
}

\begin{abstract}
KEYWORDS: Thermal Hyperspectral, LWIR (Long Wave Infrared), Constrained Energy Minimization (CEM), Minimum Noise
\end{abstract} Fraction (MNF).

\begin{abstract}
:
Airborne hyperspectral imaging is constantly being used for classification purpose. But airborne thermal hyperspectral image usually is a challenge for conventional classification approaches. The Telops Hyper-Cam sensor is an interferometer-based imaging system that helps in the spatial and spectral analysis of targets utilizing a single sensor. It is based on the technology of Fourier-transform which yields high spectral resolution and enables high accuracy radiometric calibration. The Hypercam instrument has 84 spectral bands in the $868 \mathrm{~cm}^{-1}$ to $1280 \mathrm{~cm}^{-1}$ region $\left(7.8 \mu \mathrm{m}\right.$ to $11.5 \mu \mathrm{m}$ ), at a spectral resolution of $6 \mathrm{~cm}^{-1}$ (full-width-half-maximum) for LWIR (long wave infrared) range. Due to the Hughes effect, only a few classifiers are able to handle high dimensional classification task. MNF (Minimum Noise Fraction) rotation is a data dimensionality reducing approach to segregate noise in the data. In this, the component selection of minimum noise fraction (MNF) rotation transformation was analyzed in terms of classification accuracy using constrained energy minimization (CEM) algorithm as a classifier for Airborne thermal hyperspectral image and for the combination of airborne LWIR hyperspectral image and color digital photograph. On comparing the accuracy of all the classified images for airborne LWIR hyperspectral image and combination of Airborne LWIR hyperspectral image with colored digital photograph, it was found that accuracy was highest for MNF component equal to twenty. The accuracy increased by using the combination of airborne LWIR hyperspectral image with colored digital photograph instead of using LWIR data alone.
\end{abstract}

\section{INTRODUCTION}

Remotely sensed data acquired using hyperspectral sensors contains hundreds of spectral bands acquired over contiguous wavelength range (T. M. Lillesand et al, 2004). These narrow contiguous spectral bands helps in distinguishing various types of materials. But thermal hyperspectral technique opens up new possibilities in remote sensing. These imagers acquire data in long wave infrared region $(8-12 \mu \mathrm{m})$. Using thermal infrared data it is possible to extract land surface temperature and spectral emissivity (Martin Schlerf et al, 2012). Some of the methods are already available in the literature for multispectral thermal sensors like classification method, Gray body emissivity method etc. However, it is hard to get accurate land surface temperature and emissivity easily by multispectral temperature and emissivity separation (TES) methods (José A. Sobrino et al, 2006). The usefulness of airborne LWIR hyperspectral data lies in the fact that the imagers can be flown in day as well as at night also. Mapping in this region has many advantages over visible region like detection of buried landmines and camouflage detection etc. Hence, thermal hyperspectral remote sensing technique is a powerful technique for land use/land cover applications.

In hyperspectral images there is a lot of redundancy in the data and storage problem as well. This redundancy occurs due to high correlation between the bands. This correlation decreases the classification accuracy. Now, the data volume to be processed is huge and the training samples are limited so, there occurs a phenomenon called Hughes Phenomenon. It is also called curse of dimensionality. According to this phenomenon the accuracy of classification first increases and then starts decreasing after some value. So it becomes an important task to overcome this phenomenon. To mitigate this problem we can reduce the data dimensions using data dimensionality reduction techniques like PCA, MNF, and ICA etc. This research intends to develop a methodology to use the concept of object identification from the airborne thermal hyperspectral data. The "curse of dimensionality" is dealt with data dimensionality reduction technique called minimum noise fraction MNF.

\section{DATA DIMENSIONALITY REDUCTION APPROACH: MNF (MINIMUM NOISE FRACTION)}

In hyperspectral imagery the spectral resolution is fine but as it has large number of spectral bands therefore, it increases complexity. As the number of bands in hyperspectral imagery is very large so there may be some redundant information in it. So various data dimensionality reduction approaches have been developed which reduces the number of bands without losing the information content and also segregated the noise in the data. Various techniques are minimum noise fraction MNF, principal component analysis PCA etc (Zhang lianpeng et al, 2010). Minimum noise fraction (MNF) transforms is an important technique as far as target detection is concerned. It works on the principle that it segregates noise from information content and also reduces the variance in the data. MNF transform consists of two cascaded principal components. The difference between Minimum noise fraction (MNF) transform and principal component analysis is that MNF takes into account the sensor noise and also it orders the image in terms of signal to noise ratio SNR. On the other hand PCA consider the data variance not the sensor noise.

The MNF consists of two steps. The first step transforms the data with unit variance and ensures no band to band correlation. It decorrelates the data. In the second step it applies principal component to the noise whitened data. The images are called eigen images The more large eigen vales signifies more useful information. Eigen values close to one indicates noise affected data. Now the first requirement in the process is to estimate sensor noise. Mathematically, Consider the input image matrix as

It is $\mathrm{p} \times \mathrm{N}$ matrix.

$$
\mathrm{Y}=\left[\mathrm{y}_{1}, \mathrm{y}_{2}, \ldots \mathrm{y}_{\mathrm{p}}\right]^{\mathrm{T}}
$$

Where, $p=$ number of spectral bands $\mathrm{N}=$ data length of each band

Equation 1 represents how the noise is added to signal. $\mathrm{Y}=\mathrm{S}+\mathrm{N}$

Where, $\mathrm{S}=$ Signal and $\mathrm{N}=$ Noise

Covariance matrix can be written as:

$$
\mathrm{D}(\mathrm{Y})=\Sigma=\Sigma \mathrm{S}+\Sigma \mathrm{N}
$$


MNF is a linear transform and it can be written as:

$$
\mathrm{Z}=\mathrm{ATY}
$$

\section{STEP1:}

Eigen values and Eigen vector of $\Sigma \mathrm{N}$ are found. Eigen vector matrix of $\Sigma \mathrm{N}$ is denoted by U. Make $\Sigma \mathrm{N}$ to be a diagonal matrix. The diagonal matrix is shown in equation 2 .

$$
\mathrm{DN}=\mathrm{UT} \Sigma \mathrm{N} \mathrm{U}
$$

$\mathrm{DN}$ is the eigenvalue of $\Sigma \mathrm{N}$

Now find $\mathrm{P}=\mathrm{UN}^{-1 / 2}$ such that, $\mathrm{P}^{\mathrm{T}} \Sigma \mathrm{NP}=\mathrm{I}$

Where, $\mathrm{I}=$ identity matrix.

To project original image to new space apply $\mathrm{P}$ to image data $\mathrm{Y}$ $\mathrm{X}=\mathrm{PY}$, Noise in the transformed data is unit variance.

STEP2:

The input data is transformed by using PCA i.e. principal component analysis as shown in figure 3 .

$$
\text { C D- adj }=\text { PT } \Sigma \text { P }
$$

Make C D- adj as diagonal matrix,

$$
\text { VT C D- } \operatorname{adjP}=\text { D D-adj }
$$

Where D D-adj = is a diagonal matrix and its elements are the eigenvalues of $\mathrm{C}$ D- adj. $\mathrm{V}$ is the vertical matrix of corresponding eigenvectors.

Transformed matrix is obtained, $\mathrm{A}=\mathrm{PV}$

This will arrange the data according to the SNR values. The major information is contained in first some of the MNF components and information content decreases as number of components increases. Hence, minimum noise fraction (MNF) is a linear transform which reduces the dimension, removes noise and reprojects the input data in which whole of the noise is removed which is better than PCA.

\section{TARGET DETECTION ALGORITHM}

The Constrained Energy Minimization (CEM) technique is a target detection technique which is quite popular for hyperspectral image (Chang et al, 2001). It uses a linear operator and performs matched filtering (Gass, $\mathrm{T}$ et al, 2010). It maximizes the response of the target signature and suppresses the signature of background pixels. At its background it estimates the correlation matrix for obtaining the background pixels. In short it enhances the contrast between the target and background spectra. So this is quite efficient technique especially in hyperspectral domain. In addition to its advantages it has some of the anomalies in its performance. It doesn't perform satisfactorily in presence of low probability background. In the end it produces abundance image i.e. grey level images for each class. In case of similar spectral signatures of different classes this algorithm doesn't perform well (Yin et al, 2010). Finite set of observations are

$$
\mathrm{S}=\{\mathrm{r} 1, \mathrm{r} 2, \ldots, \mathrm{rN}\}
$$

Where, $\mathrm{ri}=(\mathrm{ri} 1, \mathrm{ri} 2, \ldots ., \mathrm{riL}) \mathrm{T}$ is a input pixel vector

Desired signature $d$ is known a priori. The objective of CEM is to design a finite impulse response (FIR) linear filter with $\mathrm{L}$ filter coefficients $\{w 1, w 2, \ldots, w L\}$. The constraint $n$ the filter is that the noise should be suppressed i.e. the average output power of noise should be minimized. Equation 4 represents the constraint applied for FIR filter (Settle J, 2002).

$$
d^{T} w=\sum_{l=1}^{L} d_{l} w_{l}=1
$$

Now, the output of FIR filter is weighted sum of input image pixels which is shown in figure 5 .

$\mathrm{y}_{\mathrm{i}}=$ output of FIR filter

$y_{i}=\sum_{l=1}^{L} w_{l} r_{i l}=w^{T} r_{i}=r_{i}^{T} w$

The average output power will be as shown in figure 6 .

$$
\frac{1}{N}\left[\sum_{i=1}^{N} y_{i}^{2}\right]=\frac{1}{N}\left[\sum_{l=1}^{L}\left(r_{i}^{T} w\right)^{T} r_{i}^{T} w\right]=w^{T}\left(\frac{1}{N}\left[\sum_{i=1}^{N} r_{i} r_{i}^{T}\right]\right) w=w^{T} R_{L \times L} L^{w}
$$

So, the average output power produced by the observation set $\mathrm{S}$ and the FIR filter with coefficient vector

$$
R_{L \times L}=\frac{1}{N} \sum_{l=1}^{L} r_{l} r_{i}^{T}
$$

$\mathrm{R}_{\mathrm{L} \times \mathrm{L}}$ is the sample autocorrelation matrix of $\mathrm{S}$.

Minimizing output average power

$$
\min ^{w} \frac{1}{N} \sum_{i=1}^{N} y_{i}^{2}=\min ^{w}\left\{w^{T} R_{L \times L}{ }^{w}\right\}
$$

The optimal weight is given by:

$$
w^{*}=\frac{R_{L \times L}^{-1}}{d^{T} R_{L \times L}^{-1} d}
$$

CEM operator is given by: CEM $=\left(\mathrm{w}^{*}\right)^{\mathrm{T}} \mathrm{r}$. Value of this operator represents the estimated amount of fraction of the object signature d contained in input image pixel $r$. The image which we get from CEM filter is a grayscale image. The gray level value of each image pixel gives the detected amount of the desired object presented in the input pixels (Puckrin, E et al, 2010). After this the object detection is performed which is based on the resulting gray scale image, and classification is performed by detecting the desired objects in separate images. Due to this reason, the only required knowledge for the CEM is the desired target not all the signatures of all objects. CEM is used vastly for the detection and classification of hyperspectral images (Ren H., 2003). However, CEM is completely determined by the information used to describe the desired target signature. Thus CEM is very sensitive to the noise.

\section{TEST DATA AND STUDY AREA}

The study area for this research work has been obtained with Hyper-Cam, an airborne LWIR hyperspectral imager. The region includes a variety of natural and man-made objects. It is located in Black Lake area of Thetford Mines, province of Québec, Canada (46.047927N, 71.366893W) and the Google Earth image with the color digital photograph is shown in figure 2. The dataset used is long-wave infrared (LWIR, thermal infrared) airborne hyperspectral data which is acquired by an 84-channel imager that covers the wavelengths between 7.8 to $11.5 \mu \mathrm{m}$ with approximately $1-\mathrm{m}$ spatial resolution. The airborne LWIR hyperspectral imagery consists of 84 spectral bands in the 868 to $1280 \mathrm{~cm}^{\wedge}-1$ region $(7.8 \mu \mathrm{m}$ to $11.5 \mu \mathrm{m})$, at a spectral resolution of $6 \mathrm{~cm}^{\wedge}-1$ (full-width-half-maximum). It has been calibrated to at-sensor spectral radiance units, in W/ $\left(\mathrm{m}^{\wedge} 2 \mathrm{sr} \mathrm{cm}^{\wedge}-1\right)$

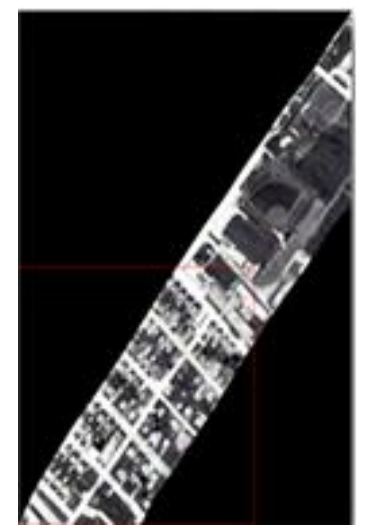

Figure 1: Airborne LWIR hyperspectral data 


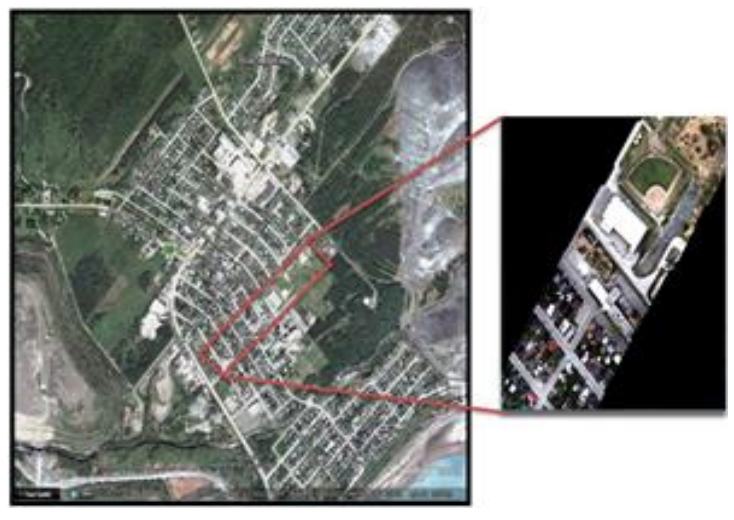

Figure 2: Google earth image of Study Area along with the digital color photograph

The average height of sensor above ground was $2650 \mathrm{ft}(807$ $\mathrm{m})$. The airborne LWIR hyperspectral imagery is shown in figure 1. Other dataset used was color digital photograph which was acquired with a digital color camera (2 Megapixel). The airborne visible imagery consists of uncalibrated, high spatial resolution, digital data with sparse ground coverage over the same area as the LWIR hyperspectral imagery. The spatial resolution of the colored digital photograph is $0.2 \mathrm{~m}$. The two airborne data sets were acquired simultaneously on May 21, 2013, between the times 22:27:36 to 23:46:01 UTC. The characteristic of the two datasets used have been mentioned in Table 1. The airborne LWIR hyperpsectral image is having 84 bands whereas, colored digital photograph has 3 bands. The LWIR imagery cover infrared region from $7.8 \mu \mathrm{m}$ to $11.5 \mu \mathrm{m}$. The average height of sensor for both the imageries is $807 \mathrm{~m}$. The spatial resolution of LWIR image is $1 \mathrm{~m}$ and for color digital photograph is $0.2 \mathrm{~m}$.

\begin{tabular}{|l|l|l|}
\hline Specifications & $\begin{array}{l}\text { Airborne LWIR } \\
\text { Hyperspectral Inage }\end{array}$ & $\begin{array}{l}\text { Colored digital } \\
\text { photograph }\end{array}$ \\
\hline No. of bands & 84 bands & 3 bands \\
\hline Spectral Bands & $\begin{array}{l}868 \text { to } 1280 \mathrm{~cm}^{\wedge}-1 \\
\text { region } \\
7.8 \mu \mathrm{m} \text { to } 11.5 \mu \mathrm{m}\end{array}$ & $\begin{array}{l}\mathrm{G} \\
\mathrm{B}\end{array}$ \\
\hline $\begin{array}{l}\text { Average height } \\
\text { of Sensor }\end{array}$ & $2650 \mathrm{ft}(807 \mathrm{~m})$ & $2650 \mathrm{ft}(807 \mathrm{~m})$ \\
\hline $\begin{array}{l}\text { Spatial } \\
\text { resolution }\end{array}$ & $1 \mathrm{~m}$ & $0.2 \mathrm{~m}$ \\
\hline
\end{tabular}

Table 1: Specification of datasets used

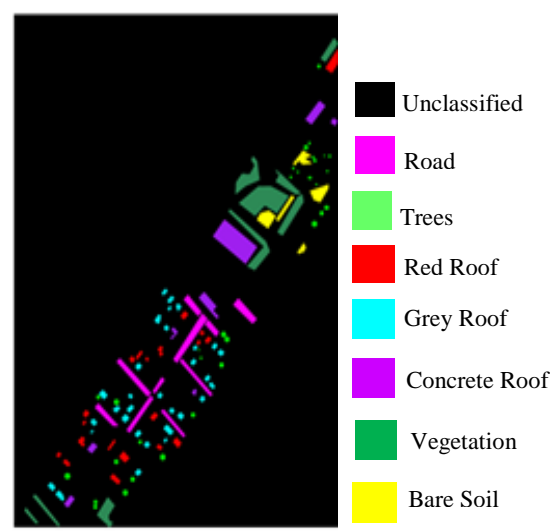

Figure 3: Ground truth image with legend

\section{METHODOLOGY ADOPTED}

The overview of the methodology adopted in this research is depicted in Figure 4. A broad division of the workflow into classification and proposed approach of dimensionality reduction (DR) and classification (each segment is explained subsequently) is illustrated and discussed in this section.

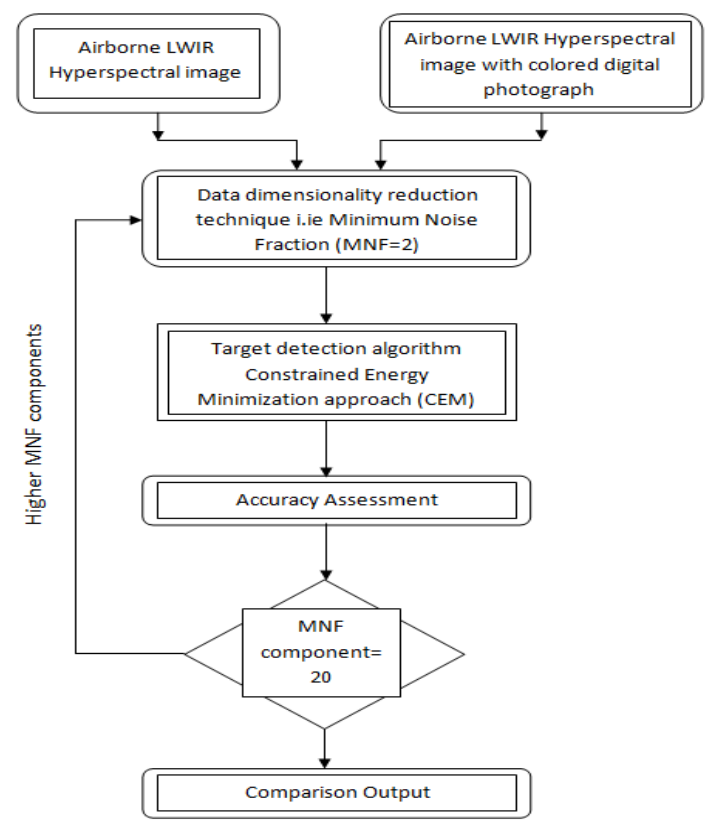

Figure 4: Methodology adopted

The overview of the methodology is that first the data set is subjected to minimum noise fraction MNF and then classification algorithm is applied to it. Firstly the airborne LWIR hyperspectral imagery is subjected to minimum noise fraction MNF which reduces the 84- bands data into a fewer component imagery and also separates the noise present in the dataset. MNF decorrelates the data using the variance matrix of LWIR data. Here it started from MNF component equal to two and then go until MNF component equal to twenty. Because accuracy starts decreasing after 20th component. These MNF components one by one go into the constrained energy minimization algorithm. Accuracy assessment is performed for each of the classified image for each and every MNF component. Then the class with highest user's accuracy is identified which means it is best classified among all classes.

Then, the similar procedure is applied to the combination of LWIR image with the colored digital photograph. For this firstly the airborne LWIR hyperspectral image is combined with the colored digital photograph. Then this image is subjected to minimum noise fraction. MNF component equal to two to twenty are given into the constrained energy minimization algorithm CEM. Accuracy assessment was conducted for each of the class. At last comparison of both the datasets is made based on the user's accuracy of each class.

\section{RESULT AND DISCUSSION}

The present research elaborates the possible effect of a classifier i.e. constrained energy minimization CEM on various number of MNF components on both the datasets. The two datasets are LWIR image and the LWIR image combined with the colored digital photograph. Firstly, the classified results of airborne LWIR hyperspectral data are shown in figure 5 to 8 and then the classified results for combination of both LWIR and color 
digital photograph are shown in figure 9 to 12 . Individual classified images using CEM (Constrained Energy Minimization) algorithm are described. The results are for MNF components equal to 20 and separate classified images for each class are shown in figure 5 to 8 . The component selection of minimum noise fraction (MNF) rotation transformation is analyzed in terms of classification accuracy using Constrained Energy Minimization (CEM) as a classifier for airborne LWIR hyperspectral image. Further all the classified images for MNF $=2$ to 20 are analyzed, through which it is interpreted that MNF component $=20$ is best for the classification purpose for airborne LWIR hyperspectral data. This has been verified by examining the user's accuracy values for each class in the classified image. Figure 5 shows results for tree and road for airborne LWIR hyperspectral image. Red roof and concrete roof are shown in figure 6, whereas figure 7 shows vegetation and bare soil. Grey roof image is shown in figure 8 .

\begin{tabular}{|c|c|c|}
\hline S.No & CLASS & $\begin{array}{c}\text { USER'S } \\
\text { ACCURACY }\end{array}$ \\
\hline 1. & Tree & $50 \%$ \\
\hline 2. & Road & $88 \%$ \\
\hline 3. & Vegetation & $55.56 \%$ \\
\hline 4. & Bare Soil & $83.34 \%$ \\
\hline 5. & Red Roof & $50 \%$ \\
\hline 6. & Concrete Roof & $42.85 \%$ \\
\hline 7. & Grey Roof & $71.72 \%$ \\
\hline
\end{tabular}

Table 2: User's accuracy of each class for MNF component 20 using CEM classifier for airborne LWIR hyperspectral image

MNF-20 is best in all MNF component groups in terms of user's accuracy which is clearly seen in table 2 . This also shows that the CEM classifier is better able to classify road with $88 \%$ accuracy, bare soil with $83.34 \%$ accuracy and grey roof with $71.72 \%$ accuracy for airborne LWIR hyperspectral data as their user accuracy is highest among all classes. Tree, vegetation and red roof shows similar accuracy with 50\%, $55.56 \%$ and $50 \%$ accuracy respectively. Concrete roof shows less than $50 \%$ accuracy i.e. $42.85 \%$.
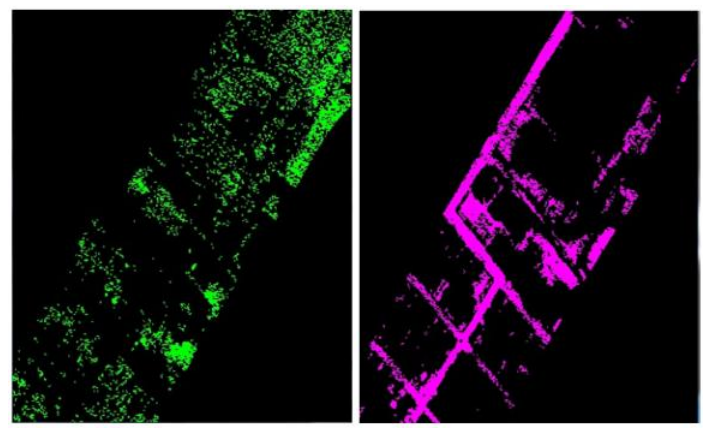

Figure 5: Classified image of individual trees (right) and classified image of road (left) using CEM (Constrained Energy Minimization) algorithm for LWIR imagery

Later, the combination of LWIR imagery and colour digital photograph is subjected to MNF and then classification is performed using CEM algorithm. Results were examined for the same. Individual classified images for each class using CEM (Constrained Energy Minimization) algorithm for MNF components equal to 20 are shown in figure 9 to 12 . The component selection of minimum noise fraction (MNF) rotation transformation is analyzed in terms of classification accuracy using Constrained Energy Minimization (CEM) as a classifier for airborne LWIR hyperspectral image.
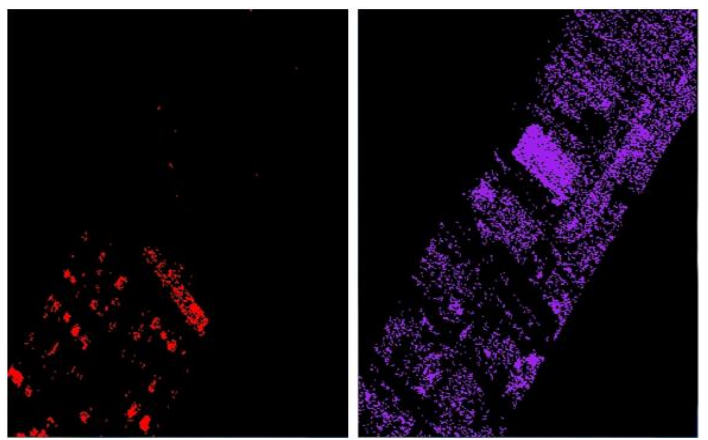

Figure 6: Classified image of red roof (right) and classified image of concrete roof (left) using CEM (Constrained Energy Minimization) algorithm for LWIR imagery
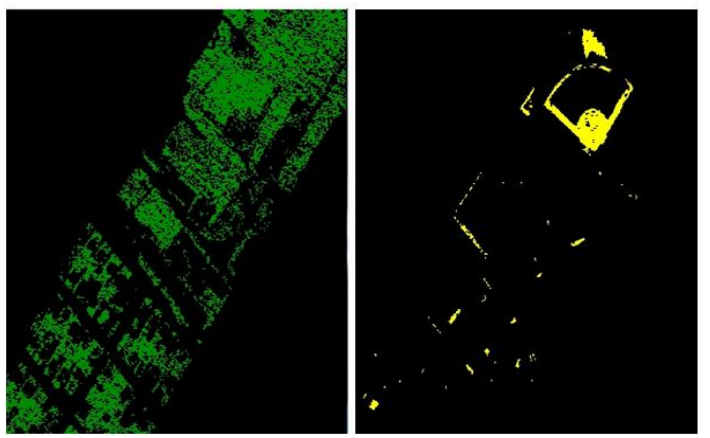

Figure 7: Classified image of vegetation (right) and classified image of bare soil (left) using CEM (Constrained Energy Minimization) algorithm for LWIR imagery

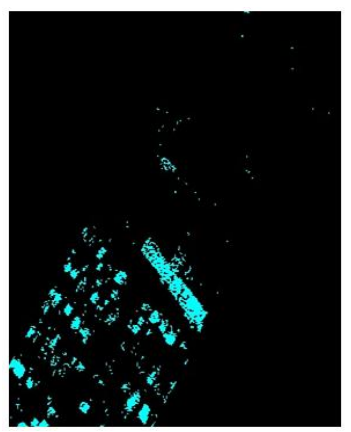

Figure 8: Classified image of grey roof (right) using CEM (Constrained Energy Minimization) algorithm for LWIR imagery

Further all the classified images for $\mathrm{MNF}=2$ to 20 are analyzed, through which it is interpreted that MNF component equal to 20 is best for the classification purpose for combination of airborne LWIR hyperspectral data with colored digital photograph. This has been verified by examining the user's accuracy values for each class in the classified image. In this also 20th MNF component reveals the highest accuracy in terms of user's accuracy for each class. Figure 9 shows results for tree and road for airborne LWIR hyperspectral image. Red roof and concrete roof are shown in figure 10, whereas figure 11 shows vegetation and bare soil. Grey roof imagery is as shown in figure 12 . 


\begin{tabular}{|c|c|c|}
\hline S.No & CLASS & $\begin{array}{c}\text { USER'S } \\
\text { ACCURACY }\end{array}$ \\
\hline 1. & Tree & $50 \%$ \\
\hline 2. & Road & $88 \%$ \\
\hline 3. & Vegetation & $62.50 \%$ \\
\hline 4. & Bare Soil & $71.42 \%$ \\
\hline 5. & Red Roof & $55.56 \%$ \\
\hline 6. & Concrete Roof & $66.60 \%$ \\
\hline 7. & Grey Roof & $83.34 \%$ \\
\hline
\end{tabular}

Table 3: User's accuracy of each class for MNF component 20 using CEM classifier for combination of LWIR with colored digital photograph
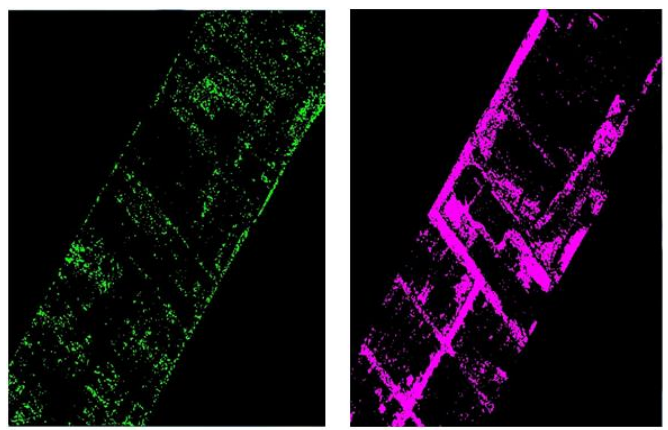

Figure 9: Classified image of individual trees (right) and classified image of road (left) using CEM (Constrained Energy Minimization) algorithm for combination of LWIR imagery with colored photograph
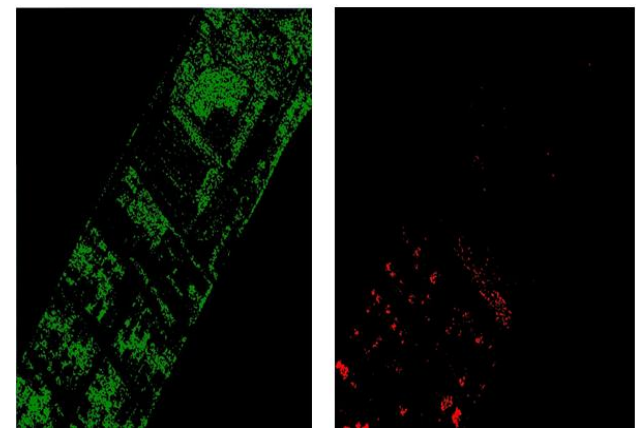

Figure 10: Classified image vegetation (right) and classified image of red roof (left) using CEM (Constrained Energy Minimization) algorithm for combination of LWIR imagery with colored photograph
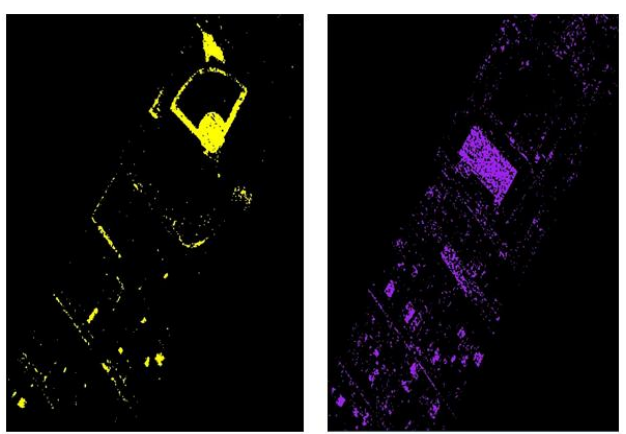

Figure 11: Classified image of bare soil (right) and classified image of concrete roof (left) using CEM (Constrained Energy Minimization) algorithm for combination of LWIR imagery with colored photograph
This approach is better able to classify three classes i.e. road, concrete roof and grey roof as their user's accuracy is highest among all classes. Table 3 explains that MNF-20 is best in all MNF component groups in terms of user's accuracy. Road, bare soil and grey roof are better classified with $88 \%, 71.42 \%$ and $83.34 \%$ accuracy. Tree and red roof are classified with similar accuracy i.e. $50 \%$ and $55.56 \%$ accuracy. Whereas, vegetation and concrete roof are classified with $62.50 \%$ and $66.60 \%$ accuracy.

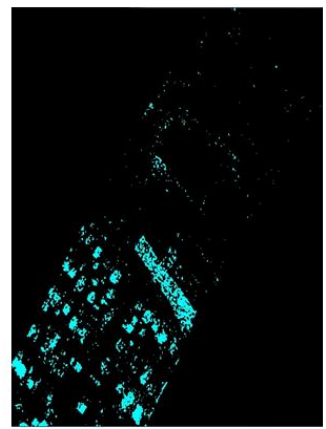

Figure 12: Classified image of grey roof (right) using CEM (Constrained Energy Minimization) algorithm for combination of LWIR imagery with colored photograph

\section{CONCLUSION}

The Airborne thermal hyperspectral image usually is a challenge for conventional classification approaches. Due to the Hughes effect, only a few classifiers are able to handle high dimensional classification task. MNF rotation is a dimensionality reducing approach to segregate noise in the data. In this, the component selection of minimum noise fraction (MNF) rotation transformation is analyzed in terms of classification accuracy using constrained energy minimization (CEM) algorithm as a classifier for Airborne thermal hyperspectral image and for the stack of LWIR and colour digital photograph. By comparing the user's accuracy obtained from the classification of airborne LWIR image and from the LWIR with colour digital photograph we can draw the inferences that there was no change in the classification of trees and road as can be deduced from the user's accuracy which is $50 \%$ for trees and $88 \%$ for the road. For some of the classes the user's accuracy increased in the LWIR with colour digital photograph. For vegetation user's accuracy increased from $55.56 \%$ to $62.50 \%$. For red roof user's accuracy increased from $50 \%$ to $55.56 \%$. For concrete roof user's accuracy increased from $42.85 \%$ to $66.67 \%$. For grey roof user's accuracy increased from $71.42 \%$ to $83.34 \%$. In contrast, the user's accuracy for one class i.e. bare soil decreased from $83.34 \%$ to $71.42 \%$ in LWIR with colour digital photograph, which means that there were some misclassifications for this class in LWIR with colour digital photograph. This result also suggests that too few MNF components (e.g. only using first 5 or 10) will result in misclassification due to bring in too little information.

Acknowledgement: The authors would like to thank Telops Inc. (Québec, Canada) for acquiring and providing the data used in this study, the IEEE GRSS Image Analysis and Data Fusion Technical Committee and Dr. Michal Shimoni (Signal and Image Centre, Royal Military Academy, Belgium) for organizing the 2014 Data Fusion Contest, the Centre de Recherche Public Gabriel Lippmann (CRPGL, Luxembourg) and Dr. Martin Schlerf (CRPGL) for their contribution of the Hyper-Cam LWIR sensor, and Dr. Michaela De Martino 
(University of Genoa, Italy) for her contribution to data preparation.

\section{REFERENCES}

Chang, C. I., Ren, H., and Chiang, S. S., 2001. Real-time processing algorithms for target detection and classification in hyperspectral imagery. Geoscience and Remote Sensing, IEEE Transactions , 39(4), pp. 760-768.

Chisense, C., Hahn, M., and Engels, J., 2012. Classification of Roof Materials Using Hyperspectral Data. AGSE , 34

Gass, T., Dreuw, P., and Ney, H., 2010. Constrained energy minimization for matching-based image recognition. In Pattern Recognition (ICPR), 20th International Conference (pp. 33043307). IEEE.

Geng, X., Ji, L., Sun, K., \& Zhao, Y., 2014. CEM: More Bands, Better Performance.

José A. Sobrino, Juan C. Jiménez-Muñoz, Pablo J. ZarcoTejada, Guadalupe Sepulcre-Cantó y Eduardo de Miguel, 2006. Land surface temperature derived from airborne hyperspectral scanner thermal infrared data. Remote Sensing of Environment, Vol. 102, pp. 99-115.

Lin, C., Wang, C. M., and Chang, C. I., 2000. Application of generalized constrained energy minimization approach to urban road detection. In Geoscience and Remote Sensing Symposium Proceedings IGARSS. IEEE, Vol. 5, pp. 2080-2082.

Lin, G. C., Wang, W. J., and Wang, C. M., 2010, August. Feature selection algorithm for classification of multispectral MR images using constrained energy minimization. In Hybrid Intelligent Systems (HIS), 10th International Conference IEEE. pp. 43-46.

Manolakis, D., Marden, D., and Shaw, G. A., 2003. Hyperspectral image processing for automatic target detection applications. Lincoln Laboratory Journal, 14(1), pp. 79-116.

Martin Schlerf, Gilles Rock, Philippe Lagueux, Franz Ronellenfitsch , Max Gerhards , Lucien Hoffmann and Thomas Udelhoven, 2012. A Hyperspectral Thermal Infrared Imaging Instrument for Natural Resources Applications, Remote Sensing, vol. 4, issue 12, pp. 3995-4009.

Puckrin, E., Turcotte, C. S., Lahaie, P., Dubé, D., Farley, V., Lagueux, P., Marcote F. and Chamberland, M., 2010, April. Airborne infrared-hyperspectral mapping for detection of gaseous and solid targets. In SPIE Defense, Security, and Sensing, International Society for Optics and Photonics, pp. 766516-766516.

Ren, H., Du, Q., Chang, C. I., and Jensen, J. O., 2003, October. Comparison between constrained energy minimization based approaches for hyperspectral imagery. In Advances in Techniques for Analysis of Remotely Sensed Data, IEEE Workshop, pp. 244-248.

Settle, J., 2002. On constrained energy minimization and the partial unmixing of multispectral images. IEEE transactions on geoscience and remote sensing, 40(3), pp. 718-721.

T. M. Lillesand, R. W. Kiefer, and J. W. Chipman, 2004. Remote Sensing and Image Interpretation, $5^{\text {th }}$ ed. Sanjeev
Offset Printers, Delhi: John Wiley and Sons (Asia) Pte. Ltd., Singapore

Yin, J., Wang, Y., Wang, Y., and Zhao, Z., 2010. A modified algorithm for multi-target detection in hyperspectral image. In Informatics in Control, Automation and Robotics (CAR), 2nd International Asia Conference, Vol. 3, pp. 105-108.

Zhang lianpeng ,Liu qinhuo , Zhao Changsheng ,Lin hui ,Sun huasheng, 2010. The detailed vegetation classification for airborne hyperspectral remote sensing imagery by combining pca and pp. Workshop on Hyperspectral Image and Signal Processing: Evolution in Remote Sensing - WHISPERS. 